\title{
Cardiovascular Disease Risk Factors and Cognition in the Elderly
}

\author{
Elizabeth C. Leritz ${ }^{1,3,4}$, Regina E. McGlinchey ${ }^{1,3,4}$, Ida Kellison², James L. Rudolph ${ }^{1,3,4}$, and \\ William P. Milberg ${ }^{1,3,4}$ \\ ${ }^{1}$ Geriatric Research, Education and Clinical Center (GRECC) \\ ${ }^{2}$ Department of Psychology, VA Boston Healthcare System \\ ${ }^{3}$ Division of Aging, Brigham \& Women's Hospital \\ ${ }^{4}$ Harvard Medical School
}

\begin{abstract}
While it is relatively widely known that cardiovascular disease (CVD) can result in cognitive decline, it is becoming increasingly clearer that actual risk factors for CVD, such as high blood pressure, diabetes, and obesity are also associated with alterations to brain structure and cognition. The prevalence of CVD risk factors increase exponentially with age and are often overlooked as a source of cognitive changes that are otherwise thought to be part of the 'normal' aging process. Associated cognitive changes are observed even at levels of risk that would be considered subclinical by current diagnostic convention, and are often significant enough to interfere with daily functional abilities. More importantly, if not controlled, CVD risk can lead to further decline, including cerebrovacsular disease and dementia. Thus, it is critically important to consider these factors in the elderly and we recommend more routine cognitive screenings, particularly when CVD risk factors are involved.
\end{abstract}

\section{Introduction}

The prevalence of cardiovascular disease (CVD) is increasing rapidly in the growing aging population. Currently, an estimated 81.1 million American adults (more than one in three) have one or more types of CVD including high blood pressure, coronary heart disease, heart failure and/or stroke. Of these, 38.1 million are 60 years of age or older [1]. The estimated direct and indirect cost of total CVD for 2010 is $\$ 503.2$ billion; as a leading cause of longterm disability in the United States [2], stroke accounts for approximately $\$ 73.7$ billion, which includes costs related to the inpatient stay, rehabilitation, and follow-up care of continuing deficits resulting from motor and cognitive impairment [1].

Although the debilitating impact of CVD and cerebrovascular disease on cognition in older adults is well-documented, perhaps less well-known is the recognition that CVD risk factors can be associated with functionally debilitating changes in cognition. Though these patients usually do not meet current diagnostic criteria for dementia, they suffer from disorders of cognitive ability that are functionally significant enough to undermine their ability to comply with the very medical treatments designed to mitigate the causative risk factors. Simply carrying a single risk factor for CVD can cause morphological alterations to brain structures [3] and if untreated can lead to more severe conditions such as cerebrovascular

Address Correspondence to: Elizabeth Leritz, Ph.D., 150 South Huntington Avenue, GRECC 182 (JP), Boston, MA 02130. bleritz@nmr.mgh.harvard.edu; Phone: 857.364.5645; Facsimile: 857.364.4544. 
disease, cognitive decline and dementia [4]. Obviously, the presence of more than one risk factor can increase these risks substantially. This realization is concerning for the maintenance of independence in the nation's older adults since, of the population between the ages of 65 and 74, 65\% of men and 70\% of women have high blood pressure; $78 \%$ of men and $70 \%$ of women are overweight or obese; and $21 \%$ of men and $19 \%$ of women are diagnosed with diabetes (AHA, 2011).

In recent years, there has been a surge of scientific research examining the impact that CVD risk factors have on cognition. The most commonly studied risk factors have been blood pressure, diabetes, and smoking, as well as metabolic-related conditions such as obesity. This article reviews the recent empirical evidence of the effect that these risk factors have on brain structure and cognition. We then discuss implications of these findings, including functional consequences as well as the importance of the management of these risk factors in preventing progression to cerebrovascular disease and dementia.

\section{Effect of CVD Risk Factors On Cognition}

Currently there is a substantial amount of evidence documenting the association between CVD risk factors and cognitive, or neuropsychological function, as well as with changes to brain structure. Studies that include examination of structural brain integrity have provided clues as to how CVD risk factors impact neuropsychological function. Currently there exists an abundant evidence of CVD-related pathological alterations in brain structure such as gray matter atrophy, increases in white matter lesions, and damage to subcortical white matter pathways, all of which have been correlated with decrements on neuropsychological tests [3, 5]. In a recent review article, Flicker described ten CVD risk factors that may impact the brain, each via distinct yet overlapping vascular or cellular-related mechanisms, highlighting the broad impact that CVD risk can have on brain structure and function [6].

In the neuropsychological literature on CVD risk, blood pressure (BP) is perhaps the most widely studied factor. Associations between high blood pressure (BP) and poor performance on neuropsychological tests are well described, particularly in the areas of memory, attention, and executive function, a domain that involves higher order cognitive processes such as reasoning, planning, cognitive flexibility, and initiation of appropriate behavior [3]. This was most recently demonstrated in a study that examined the relationship between BP and cognition in 71 individuals with normal BP and 63 individuals with chronically high BP [7]. Participants were classified according to age (younger, middle and older) in order to examine how BP specifically impacts executive function and processing speed across the lifespan. While composite measures of executive function and perceptual speed were reduced across age groups, the high $\mathrm{BP}$ group exhibited a steeper decline, independent of age, education, or sex. Evidence of the BP-cognition relationship was also supported by a recent paper reporting that higher diastolic $\mathrm{BP}$ was associated with poor cognition in a large sample of nearly 20,000 older adults [8]. These recent results confirm previous findings in the literature, and provide additional support for the idea that CVD risk factors such as high BP impact cognition, but may have a specific deleterious impact on executive functioning.

Longitudinal studies provide conclusive data indicating that persistent presence of CVD risk factors places individuals at dramatically increased risk for stroke and other serious vascular consequences. For example, Oveisgharan and Hachinski (2010) examined the relationship between hypertension and cognition over a period of 11 years in a sample of 990 participants with a range of CVD risk and cognitive ability [9]. Hypertension did not appear to contribute to overall cognitive decline in those individuals who progressed to dementia. However, in hypertensive subjects, the presence of executive, but not memory, dysfunction at baseline was associated with a more rapid development of dementia symptoms, supporting a potential interaction between high blood pressure and cognition. Consistent 
with this idea that high BP negatively affects executive function specifically, Wiberg et al. (2010) reported that poorer performance on a hallmark measure of executive function (Trailmaking part B) was significantly predictive of future stroke or TIA, even when controlling for education, social group, and other CVD risk factors [10].

Metabolic-related conditions such as diabetes and obesity have also been associated with cognitive impairment. Rasgon et al. (2009) studied insulin resistance (IR), a condition that is believed to underlie many vascular-based disorders including diabetes, and obesity. They examined the impact that IR has on brain structure (specifically, hippocampal volume) and cognition in a sample of middle-older aged women who were on hormone replacement therapy [11]. Results revealed that independent of hormone therapy or exposure, higher IR was associated with smaller volume of the left and right hippocampus and with worse performance on cognition, particularly on tests of learning and memory. Not only do these findings support a structure-function correlation between the hippocampus and memory, but they are also consistent with emerging theories that diabetes-related conditions may have a selective impact on posterior brain structures involved in memory, and as the authors suggest, may be a biomarker of increased risk for dementia syndromes such as Alzheimer's disease. A recent study confirmed the impact that diabetes has on brain structure and cognition; in a study examining aging and cognition in a community-dwelling sample of middle and older-aged adults, patients with diabetes had smaller overall brain volume as well as more profound deficits on measures of attention and working memory [12]. Longitudinal studies suggest that the rates of cognitive decline as well as physical and functional decline are increased in older adults with type 2 diabetes [13] [14]. Moreover, a recent meta-analysis reported an increased risk of dementia in patients with diabetes compared to those without [15], supporting the idea that the presence of CVD risk factors may represent a precursor to cognitive decline.

Obesity, which is related to various health-related conditions, including diabetes, has also been found to have a negative effect on both brain structure and cognition. Walther et al. examined how body mass index (BMI), an indicator of obesity and metabolic function, related to brain structure and neuropsychological function in a group of 95 communitydwelling older females [16]. As predicted, higher BMI was significantly correlated with reduced gray matter volumes in several brain regions, as well as with poorer performance, specifically on measures of executive function. Smaller volume of the left orbitofrontal region, a brain area that is known to underlie complex cognitive tasks, was also associated with poorer executive function, thus establishing a mediation cascade whereby CVD risk (obesity) impacts brain structure and subsequent cognition. Importantly, controlling for hypertension did not alter any of the effects, indicating that obesity may have a negative impact on the brain in and of itself, and therefore should be considered a potentially significant risk factor for the development of further disease and cognitive decline. In a large-scale epidemiological study, Fitzpatrick et al. (2009) examined how body mass index (BMI) contributed to the development of dementia in nearly 3000 individuals participating in a longitudinal study of cardiovascular health (The Cardiovascular Health Study; CHS) that included administration of a comprehensive battery of neuropsychological tests for the purpose of diagnosing dementia [17]. BMI measurements were taken from two age groups in the study; middle age (age 50 years) and late life (65 years or older). While greater BMI in midlife was associated with a significantly higher risk of dementia, obesity in late life was inversely related such that underweight individuals had a higher incidence of dementia diagnosis. This paradoxical finding has been reported before [18], and it has been suggested that in later life, low weight is often associated with conditions such as frailty and poor nutrition, which by themselves are risk factors for cognitive decline. However, the finding of increased dementia risk in middle age supports the idea that it may be the pre-aging 
exposures to such CVD risk factors as obesity that are the most critical to manage, and may present the greatest risk for the development of dementia.

Smoking is an additional risk factor that by itself can pose a significant risk to the cardiovascular and cerebrovascular system, but is often examined in combination with other CVD risk factors. In addition to its role as an independent predictor of cardiovascular morbidity and mortality, smoking has been associated with accelerated cerebral atrophy, increased white matter lesions and cerebral hypoperfusion, as well as reduced cortical grey matter density in several brain regions that are also associated with AD [19]. Even in middle-aged adults, smoking is related to cognitive deficits including reduced psychomotor speed and cognitive flexibility [20]. Moreover, although results of early case control studies suggested that smoking was protective for the development of Alzheimer's disease, recent systematic reviews of longitudinal studies report that current smoking increases the risk of Alzheimer's disease and may increase the risk of other dementias in older adults, particularly in combination with other CVD risk factors [21].

Additional risk factors such as high cholesterol, and other cardiovascular-related conditions have been studied less in isolation. However, these factors are often included in papers examining the impact of multiple CVD risk factors on cognition. These studies are also important, as a high percentage of the adult population typically presents with more than one risk factor. Recently, a large-scale longitudinal study of middle-older aged adults found that of a host of risk factors (hypertension, diabetes, hyperlipidemia, smoking, and carotidintima-media wall thickness), only hypertension and diabetes were associated with cognitive decline over a 6-year period [22]. In a more recent longitudinal study, Wendell et al. (2009) found that greater carotid intimal medial thickness, a potential measure of subclinical vascular disease, was associated with greater decline on neuropsychological tests of memory and executive function, even when controlling for a host of demographic and other CVD risk factors including age, education, gender, BP, BMI, cholesterol, smoking risk, depression and medication use [23]. Importantly, these findings were reported in a sample of individuals with no cardiovascular or cerebrovascular disease, indicating a potential biomarker for the development of cognitive decline in subclinical CVD.

More formal attempts to understand and characterize syndromes that involve multiple risk factors have also been undertaken. The term 'metabolic syndrome' has been recently introduced as a term to describe a collection of CVD risk factors, and has been associated with cognitive decline, particularly in aging. In 2009, Yaffe et al. conducted a prospective four-year study of metabolic syndrome, defined according to the National Cholesterol Education Program Third Adult Treatment Panel guidelines, which suggest the presence of metabolic syndrome if 3 or more of the following symptoms are present: 1) high BMI, 2) high triglycerides, 3) low HDL, 4) hypertension or currently on antihypertensive medication, or 5) elevated fasting glucose level [24]. Of the nearly 5000 high-functioning, communitydwelling women followed (mean age 66 years), $10.2 \%$ had metabolic syndrome, and 7.2\% developed cognitive impairment and dementia [24]. Additionally, the authors report that an increase in the number of metabolic syndrome components (listed above) was also associated with a $23 \%$ age-adjusted increase in risk for dementia, and high glucose was the only risk factor with an independently higher risk than the collective syndrome. These results not only highlight the importance of managing CVD risk factors prior to any manifestation of cognitive decline, but also of considering the potential impact of multiple risk factors, such as those that constitute the metabolic syndrome.

It is also important to consider the fact that there are certain genetic factors that may predispose an individual to be more vulnerable to the impact of CVD risk factors. For example, in recent years, a link has been established between genetic variants of the 
apolipoprotein epsilon 4 allele, a known risk factor for the development of Alzheimer's disease, and the manifestation of cardiovascular and cerebrovascular disease. A recent study of the Framingham Offspring Cohort examined how Framingham Stroke Risk Profile (FSRP) scores related to measures of neuropsychological function and brain structure, and how the presence of the apoeE4 allele modifies these relationships [25]. FSRP is a composite measure of CVD risk factors that includes age, systolic blood pressure, use of antihypertensive medication, cholesterol, smoking status, diabetes, history of cardiovascular disease, the presence of left ventricular hypertrophy, and atrial fibrillation. Consistent with the idea that higher risk impacts both brain structure and cognition, the authors report significant associations between higher FSRP score and poorer performance on measures of memory and executive function. However, these relationships were moderated by the presence of the apoE4 such that they were only significant in the group that possessed the allele; further analyses with individual risk factors revealed that systolic BP appeared to be driving the effect. Moreover, higher FSRP score was associated with reduced volume of several brain structures including the frontal lobes, temporal lobes, temporal horn, and lateral ventricles, supporting the idea that the detrimental effect that elevated CVD risk has on cognition is likely driven in part by alterations to underlying brain structures. Taken together with prior studies describing interactions among apoE4, CVD risk, brain structure, and cognition, these recent findings highlight the importance of considering multiple variables when evaluating the impact of CVD risk.

\section{The Impact of Subclinical CVD Risk on Cognition}

It is now becoming more apparent that levels of CVD risk factors, which are below threshold for a clinical diagnosis such as hypertension, may have a negative impact on brain structure and cognition, albeit on a much subtler level. Recently, our laboratory examined how blood pressure relates to white matter integrity and cognition in a sample of 52 middleolder aged African Americans, a racial group with an increased susceptibility to CVD and its effects [26]. Importantly, all participants were neurologically healthy with no history of cerebrovascular disease, and represented a range of BP levels that varied from mild (i.e., no risk) to moderate/severe (i.e., high risk). We found that even in mild risk levels, higher BP was related to lower indices of white matter miccrostructural integrity in the anterior corpus callosum, and on a whole brain level; these brain regions are consistent with prior findings that BP may have a selective effect on anterior brain areas. Although we did not find any significant relationships between BP and cognition, the correlation between BP and neuropsychological function approached significance, such that higher BP was associated with poorer performance on measures of executive function. These findings are important, and suggest that across a wide range of values, BP may have a negative effect on brain structure. While relationships with cognition only approached significance, we suggest that it is possible that these become more salient with time, and that the evidence of a relationship with brain structure is an early sign of risk for cognitive decline. This would highlight the importance of aggressively monitoring and managing CVD risk factors in both middle- and older-aged adults in order to prevent progression as much as possible. The concept of subclinical risk and its effect on brain structure and cognitive function has been examined by other recent studies [27], including a recent study that reported that pulse pressure and high glucose levels were associated with poorer cognitive performance in healthy adults [28]. We recently published a study from our laboratory that demonstrated a range of values of CVD risk factors, including BP, cholesterol, and diabetes, were associated with alterations to the thickness of the human cerebral cortex [29]. Importantly, these associations were present in values that are below the current clinical cutoffs for disease, and occurred in several risk factors (i.e., hypertension, cholesterol, and glucose) that normally occur in aging. Collectively, these findings highlight the importance of considering how subclinical levels may impact cognition. 


\section{Functional Implications of the Effect of CVD Risk on Cognition}

There is substantial empirical evidence that CVD risk factors impact cognition; however, there is less research on the functional significance of these deficits, though this may perhaps be the most important from a clinical standpoint. Indeed, some studies have reported that cognitive impairments resulting from CVD risk factors affect independent activities of daily living (IADLs). For example, in the study previously reported by Christman et al. (2010), the cognitive deficits associated with diabetes were also significantly related to decrements in IADLS [12]. Our own clinical experiences are consistent with what has been reported; we find that CVD risk factors such as high blood pressure, high cholesterol, diabetes, or smoking history often display a pattern of cognitive deficits consistent with mild or moderate executive dysfunction. Often, such patients also have evidence of subclinical white matter disease seen on MRI scans. Functionally, these patients may report symptoms such as forgetting names and lists, word-finding difficulties, and misplacing things, and it is also common to observe circumstances in which IADLs such as medication or treatment adherence, bill paying or shopping are adversely affected. Executive deficits that are related to subclinical CVD can therefore have far-reaching implications, and have been touted as a major determinant of disability [30, 31]. Thus their identification and management may be critical for preventing disability.

Unfortunately, it is not unusual for such symptoms to be minimized as signs of 'normal' aging. In fact, many of the subtle cognitive impairments that are reported in conjunction with CVD risk are also reported in studies describing cognitive changes in "healthy" aging. A review of the cognitive aging literature suggests that conditions such as high blood pressure, high cholesterol, and diabetes are vastly underrepresented, despite the fact that their prevalence increases so dramatically in the growing aging population. One reason for this underrepresentation is the fact that many studies rely on self-report without clinical confirmation, and many of these conditions are under diagnosed. In our experiences, the underrepresentation in the scientific world may also be reflected by a tendency in the medical community to fail to consider subtle cognitive signs in routine physical examinations. Often times, cognitive changes are not given much attention until they reach a level of more severe functional disturbance. We suggest that subtle cognitive impairment, in and of itself, is a risk factor, and combined with one or more CVD risk factors such as high blood pressure or diabetes, represents a significant risk for cognitive decline and dementia or more severe vascular consequences such as stroke. While there has been a considerable amount of effort in the past decade to identify preclinical dementia states (such as mild cognitive impairment, considered to be prodromal Alzheimer's disease), we recommend that more routine cognitive screenings begin as early as middle age. It may be that consideration of CVD risk is something that should constitute examination of the 'normal' cognitive aging process, and that variation in risk factors is a more standard feature of age-related cognitive decline than was once thought.

Such screenings have become more possible by the development of cognitive instruments that take minimal time to administer. Brief screening measures such as the Mini Mental State Examination [32], and the Modified Mini-Mental State (3MS) Examination [33] offer broad gauges of an individual's cognitive functioning, especially when used over time so as to observe multiple data points. Our group has developed an instrument called the "Clock in the Box," which involves a very brief (less than 5 minutes) screen of memory and executive functioning, two of the most commonly affected domains in CVD risk and dementia [34]. The CIB has proven utility in a variety of clinical populations, including diabetes [35], and we have found it to be very predictive of daily function in geriatric patients. We recommend that measures such as the CIB be integrated into regular physician check-ups with patients, particularly those who have high blood pressure, high cholesterol or diabetes. 


\section{Conclusions}

In summary, CVD risk factors such as high blood pressure, diabetes, and obesity appear to have broad impact on brain structure and cognition. While impairments have been reported in many domains, executive function and memory appear to be the most commonly cited, and these findings are supported by evidence of structural alterations in specific brain regions. More recently, associations among risk factors, brain structure, and function have been reported in conjunction with subclinical levels of risk, raising awareness of the fact that even subtle cognitive changes that are thought to be reflective of the normal aging process may in fact be a symptom of CVD risk. Importantly, although cognitive changes associated with CVD risk factors may be milder and not severe enough to warrant a clinical diagnosis of dementia, their detection and management is just as critical in order to prevent worsening of symptoms. We advocate for regular screenings of cognitive abilities beginning as early as middle age, as the detection of even subtle changes, particularly in the context of CVD risk factors, may assist greatly in the prevention of dementia, thereby having a critical impact on a growing problem in our current healthcare system.

\section{Acknowledgments}

This work was supported by a grant from the National Institute of Neurologic Disorders and Stroke (K23NS062148) and by Medical Research Service VA Merit Review Awards to William Milberg and Regina McGlinchey.

\section{References}

1. American Heart Association. 2011

2. Morbidity and Mortality Weekly Report. Vol. 50. 2001. p. 120-125.

3. Raz N, Rodrigue KM. Differential aging of the brain: patterns, cognitive correlates and modifiers. Neurosci Biobehav Rev. 2006; 30(6):730-48. [PubMed: 16919333]

4. Kivipelto M, et al. Apolipoprotein E epsilon4 allele, elevated midlife total cholesterol level, and high midlife systolic blood pressure are independent risk factors for late-life Alzheimer disease. Ann Intern Med. 2002; 137(3):149-55. [PubMed: 12160362]

5. Hajjar I, et al. Hypertension, white matter hyperintensities, and concurrent impairments in mobility, cognition, and mood: the cardiovascular health study. Circulation. 123(8):858-65. [PubMed: 21321150]

**6. Flicker L. Cardiovascular risk factors, cerebrovascular disease burden, and healthy brain aging. Clin Geriatr Med. 2010; 26(1):17-27. [PubMed: 20176290] This is an important review article which discusses the relationship between common cardiovascular risk factors, including hypertension, smoking, high cholesterol, and diabetes, and cognitive function. The author discusses the importance of interventions, despite inconclusive studies in the literature, as they can potentially positively affect brain function.

*7. Bucur B, Madden DJ. Effects of adult age and blood pressure on executive function and speed of processing. Exp Aging Res. 2010; 36(2):153-68. [PubMed: 20209419] This study found that while age was associated with generalized declines in executive function and perceptual speed, high blood pressure (BP) contributed to additional deficits in executive function. This demonstrates that in older adults, higher BP may have an enhanced effect on cognition.

8. Tsivgoulis G, et al. Association of higher diastolic blood pressure levels with cognitive impairment. Neurology. 2009; 73(8):589-95. [PubMed: 19704077]

9. Oveisgharan S, Hachinski V. Hypertension, executive dysfunction, and progression to dementia: the canadian study of health and aging. Arch Neurol. 67(2):187-92. [PubMed: 20142526]

10. Wiberg B, et al. Cognitive function and risk of stroke in elderly men. Neurology. 74(5):379-85. [PubMed: 20124202]

11. Rasgon NL, et al. Insulin resistance and hippocampal volume in women at risk for Alzheimer's disease. Neurobiol Aging. 2009 
12. Christman AL, et al. Cranial volume, mild cognitive deficits, and functional limitations associated with diabetes in a community sample. Arch Clin Neuropsychol. 2010; 25(1):49-59. [PubMed: 19942595]

13. Allen KV, Frier BM, Strachan MW. The relationship between type 2 diabetes and cognitive dysfunction: longitudinal studies and their methodological limitations. Eur J Pharmacol. 2004; 490(1-3):169-75. [PubMed: 15094083]

14. Kuo HK, et al. Effect of blood pressure and diabetes mellitus on cognitive and physical functions in older adults: a longitudinal analysis of the advanced cognitive training for independent and vital elderly cohort. J Am Geriatr Soc. 2005; 53(7):1154-61. [PubMed: 16108933]

15. Cukierman T, Gerstein HC, Williamson JD. Cognitive decline and dementia in diabetes-systematic overview of prospective observational studies. Diabetologia. 2005; 48(12):2460-9. [PubMed: 16283246]

16. Walther K, et al. Structural brain differences and cognitive functioning related to body mass index in older females. Hum Brain Mapp. 2009

17. Fitzpatrick AL, et al. Midlife and late-life obesity and the risk of dementia: cardiovascular health study. Arch Neurol. 2009; 66(3):336-42. [PubMed: 19273752]

18. Curtis JP, et al. The obesity paradox: body mass index and outcomes in patients with heart failure. Arch Intern Med. 2005; 165(1):55-61. [PubMed: 15642875]

19. Almeida OP, et al. Smoking is associated with reduced cortical regional gray matter density in brain regions associated with incipient Alzheimer disease. Am J Geriatr Psychiatry. 2008; 16(1): 92-8. [PubMed: 18165464]

20. Kalmijn S, et al. Cigarette smoking and alcohol consumption in relation to cognitive performance in middle age. Am J Epidemiol. 2002; 156(10):936-44. [PubMed: 12419766]

21. Barnes DE, et al. Secondhand smoke, vascular disease, and dementia incidence: findings from the cardiovascular health cognition study. Am J Epidemiol. 2010; 171(3):292-302. [PubMed: 20051462]

22. Knopman D, et al. Cardiovascular risk factors and cognitive decline in middle-aged adults. Neurology. 2001; 56(1):42-8. [PubMed: 11148234]

23. Wendell CR, et al. Carotid intimal medial thickness predicts cognitive decline among adults without clinical vascular disease. Stroke. 2009; 40(10):3180-5. [PubMed: 19644063]

24. Yaffe K, et al. The metabolic syndrome and development of cognitive impairment among older women. Arch Neurol. 2009; 66(3):324-8. [PubMed: 19273750]

25. Zade D, et al. Interactive effects of apolipoprotein E type 4 genotype and cerebrovascular risk on neuropsychological performance and structural brain changes. J Stroke Cerebrovasc Dis. 2010; 19(4):261-8. [PubMed: 20471857]

$* * 26$. Leritz EC, et al. Variation in blood pressure is associated with white matter microstructure but not cognition in African Americans. Neuropsychology. 2010; 24(2):199-208. [PubMed: 20230114] This is one of the first studies to systematically examine how common CV risk factors (blood pressure, cholesterol, and glucose) related to brain structure. This study demonstrates that even subclinical levels of these factors are related to alterations in specific brain regions.

27. Kennedy KM, Raz N. Pattern of normal age-related regional differences in white matter microstructure is modified by vascular risk. Brain Res. 2009; 1297:41-56. [PubMed: 19712671]

28. Dahle CL, Jacobs BS, Raz N. Aging, vascular risk, and cognition: blood glucose, pulse pressure, and cognitive performance in healthy adults. Psychol Aging. 2009; 24(1):154-62. [PubMed: 19290746]

29. Leritz EC, et al. Thickness of the human cerebral cortex is associated with metrics of cerebrovascular health in a normative sample of community dwelling older adults. Neuroimage. 2011; 54(4):2659-71. [PubMed: 21035552]

30. Insel K, et al. Executive function, working memory, and medication adherence among older adults. J Gerontol B Psychol Sci Soc Sci. 2006; 61(2):P102-7. [PubMed: 16497953]

31. Royall DR, Chiodo LK, Polk MJ. Correlates of disability among elderly retirees with "subclinical" cognitive impairment. J Gerontol A Biol Sci Med Sci. 2000; 55(9):M541-6. [PubMed: 10995053] 
32. Folstein MF, Robins LN, Helzer JE. The Mini-Mental State Examination. Arch Gen Psychiatry. 1983; 40(7):812. [PubMed: 6860082]

33. Teng EL, Chui HC. The Modified Mini-Mental State (3MS) examination. J Clin Psychiatry. 1987; 48(8):314-8. [PubMed: 3611032]

**34. Grande LJ, et al. Detecting cognitive impairment in individuals at risk for cardiovascular disease: the "Clock-in-the-Box" screening test. Int J Geriatr Psychiatry. This article reports on a brief screening measure for cognitive function, the Clock-in-the-Box (CIB). The CIB was found to have good reliability and predictive validity in a population with cardiovascular risk factors. The CIB is a potentially very useful screening tool for clinicians.

35. Munshi M, et al. Cognitive dysfunction is associated with poor diabetes control inolder adults. Diabetes Care. 2006; 29(8):1794-9. [PubMed: 16873782] 